\title{
A Seemingly Universal Particle Kinetic Distribution in Porous Media
}

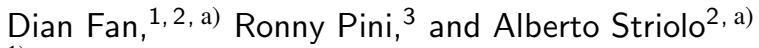 \\ ${ }^{1)}$ School of Environmental Science and Engineering, Southern University of Science and Technology, Shenzhen 518055, \\ China. \\ ${ }^{2)}$ Department of Chemical Engineering, University College London, London WC1E 7JE, \\ $U K$. \\ ${ }^{3)}$ Department of Chemical Engineering, Imperial College London, London SW7 2AZ, UK.
}

(Dated: 7 September 2021)

We study many-particle transport in randomly jammed packing of spheres at different particle Péclet numbers $\left(P e^{*}\right)$. We demonstrate that a modified Nakagami- $m$ function describes particle velocity probability distributions when particle deposition occurs. We assess the universality of said function through comparison against new Lagrangian simulations of various particle types as well as experimental data from the literature. We construe the function's physical meaning as its ability to explain particle deposition in terms of $P e^{*}$ and the competition between distributions of energy barriers for particle release and particles' diffusive energy.

Many-particle systems in porous media are ubiquitous in Nature, yielding a wealth of important phenomena ranging from clustering in granular gases ${ }^{1}$, localization transition during colloidal gelation ${ }^{2}$, biofilms formation ${ }^{3}$, solute dispersion $^{4}$ as well as nanoparticles' deposition in porous media $^{5-7}$. Understanding the particles' kinetic probability distribution in real porous media is essential to achieve particulate control $^{8,9}$. Prior studies described how such distributions depend on pore structural variables (e.g., obstacle size $^{10}$, porosity ${ }^{11-13}$, pore structure ${ }^{14}$ ) by fitting the probability data into standard probability distribution functions (PDFs), such as exponential ${ }^{10,15,16}$, stretched exponential $^{11,17}$, power-law $^{14,18}$, and power-exponential ones ${ }^{12}$. However, the assessment of their predictive ability remains elusive, because these functions contain fitting parameters that often lack a solid physical foundation.

Phenomenologically, laboratory particle-tracking data for solutes, nanoparticles (NPs), and microparticles (MPs) through random packs of spheres manifest non-Gaussian velocity probability distributions $3,10,19,20$, indicating that the fluid velocity is in general different from the particle transport velocity $^{21}$. The similarity among these distributions further suggests that a universal function might be able to describe particle kinetics. Should such universal function exist, it should be consistent with rigorous mathematical derivations, such as the Maxwell-Boltzmann (MB) distribution when real particles can be regarded as ideal gas particles ${ }^{22}$. The former condition holds when particles are not interacting with each other nor with porous media. In porous media, however, particles travel complex paths that are largely governed by local fluctuations in the velocity field of the fluid that carries them, in addition to other mechanisms, such as particle deposition ${ }^{23}$ and exclusion effects ${ }^{24}$, in which case the MB distribution is likely to break down ${ }^{25}$. This observation calls for new theoretical developments to describe statistical particles' kinetics in porous media.

In this Letter, we study the transport of three typical types of particle ensembles - solutes, NPs, and MPs, in an incom-

\footnotetext{
a) Authors to whom correspondence should be addressed:

Dian Fan, fand@sustech.edu.cn; Alberto Striolo, a.striolo@ucl.ac.uk
}

pressible, viscous, laminar fluid flowing through a rigid heterogeneous medium at steady-state under a constant pressure gradient. The medium is obtained via a three-dimensional, randomly jammed pack of spheres; the particles, whose sizes are much smaller than the average pore size, transport advectively following the heterogeneous flow field generated by the variation of pore structures, ranging from micropores $\left(>10^{-6}\right.$ m) to connected pore networks $\left(\sim 10^{-3} \mathrm{~m}\right)$; for particles transported near the pore walls, e.g., within $\sim 100 \mathrm{~nm}\left(<10^{-7} \mathrm{~m}\right)$ from the walls, advective transport becomes negligible and diffusion dominates; particles may deposit if the particle-wall electrostatic interactions are sufficiently attractive; particleparticle interactions are not considered in our model. We derive a seemingly universal function able to describe the particles' velocity distribution in the medium by modeling particles' behaviors near the pore center and the pore wall, respectively. The model proposed rationalizes that particle Péclet number and the strength of particle-wall interactions govern particle kinetic distributions.

Let us consider the longitudinal transport of particles driven by a pressure gradient $(\nabla P)$ in the porous medium. Particles near the pore center are unaffected by particle-wall interactions because studied particle sizes are much smaller than the average pore diameter. Under such conditions, the particle longitudinal velocity $\left(v_{\mathrm{pL}}\right)$ can be decomposed into advective $\left(u_{\mathrm{pL}}\right)$ and diffusive components $\left(w_{\mathrm{pL}}\right)$, i.e., $v_{\mathrm{pL}}=u_{\mathrm{pL}}+w_{\mathrm{pL}}$. We consider a wide range of the particle Péclet numbers $P e^{*}=\left\langle v_{\mathrm{w}}\right\rangle d_{\mathrm{p}} / \mathcal{D}_{\mathrm{w}}{ }^{26}$ (where $\left\langle v_{\mathrm{w}}\right\rangle$ is the mean ambient fluid velocity; $d_{\mathrm{p}}$ is particle size; $\mathcal{D}_{\mathrm{w}}$ is molecular diffusion coefficient of the ambient fluid): when $P e^{*}<<1$, the mean magnitude of velocities $\left\langle v_{\mathrm{pL}}\right\rangle\left\langle\left\langle w_{\mathrm{pL}}\right\rangle\right.$; when $P e^{*}>1,\left\langle v_{\mathrm{pL}}\right\rangle>\left\langle w_{\mathrm{pL}}\right\rangle$. The correspondent (longitudinal) total energy of each particle is $E=m_{\mathrm{p}} v_{\mathrm{pL}}^{2} / 2^{27}$.

Due to the imposed $\nabla P$, particles that transport in the fluid at location $x$ experience an energy contributed by a flow potential $\varphi(x)=-\nabla P x / \bar{n} m_{\mathrm{p}}{ }^{28}$, where $\bar{n}$ is the mean particle number density, and $m_{\mathrm{p}}$ is the mean particle mass. The flow potential here is the mechanical energy required to move a fluid mass unit from a reference position to the position of interest at a constant velocity ${ }^{29}$. Note that $x$ defines positions within the porous matrix rather than the instantaneous position nor displacement of the particles (see SM for details). To 
model the local particle number density $n(x)$ in the flow potential field, we consider the Boltzmann distribution because of the distribution's applicability to an external space-dependent potential field ${ }^{25}$ (e.g., gravitational ${ }^{28}$ or electric fields ${ }^{30}$ ): At time $t \rightarrow+\infty$, (i.e., the equilibrium state in the flow potential is reached $\left.{ }^{28}\right), n(x)$ obeys the Boltzmann distribution as a function of $\varphi(x): n(x)=n(x=0) \times \exp \left(\nabla P x / \bar{n} k_{\mathrm{B}} T\right)$, where $k_{\mathrm{B}}$ is the Boltzmann constant and $T$ is the absolute temperature.

By coupling $n(x)$ with the local MB distribution ${ }^{31}$, we derive (see Supplementary Material (SM), Sec. II) the velocity PDF for particles near the pore center:

$$
f_{V}\left(v_{\mathrm{pL}}\right) \propto \exp \left(-\frac{-\frac{\nabla P}{\bar{n}} x+\frac{1}{2} m_{\mathrm{p}} w_{\mathrm{pL}}^{2}}{k_{\mathrm{B}} T_{1}}\right) \propto \exp \left(-\frac{m_{\mathrm{p}} v_{\mathrm{pL}}^{2}}{2 k_{\mathrm{B}} T_{1}}\right),
$$

where $T_{1}$ is the temperature that reflects the mean $\langle E\rangle=$ $m_{\mathrm{p}}\left\langle v_{\mathrm{pL}}^{2}\right\rangle / 2=k_{B} T_{1} / 2$. Equation (1) considers that after the particles are introduced at the upstream of the porous medium (at $x=0$ ) with zero velocity, their flow potential decreases as the advective kinetic energy increases, i.e., $\quad-\nabla P x / \bar{n}=$ $m_{\mathrm{p}} u_{\mathrm{pL}}^{2} / 2$.

For particles near the pore wall (i.e., obstacle surfaces), it is assumed that advective velocity is negligible (i.e., $u_{\mathrm{pL}}=0$ ) because of fluid stagnation. The particles' longitudinal velocity near the pore wall $\left(v_{\mathrm{pL}}\right)$ is the sum of the diffusive velocity $\left(w_{\mathrm{pL}}\right)$ and an additional velocity term $(\theta)$ affected by the particle-wall interactions: $v_{\mathrm{pL}}=w_{\mathrm{pL}}+\boldsymbol{\theta}$. To quantify such particle-wall interactions, we adopt the extended DerjaguinLandau-Verwey-Overbeek (XDLVO) formalism, where the net interaction energy is due to the combination of Londonvan der Waals, electric double layer, and short-range Born interactions ${ }^{32}$. A typical XDLVO curve of net interaction energy versus separation distance features a deep primary minimum and a shallow secondary minimum, separated by a repulsive maximum (see SM, Sec. II). The difference between the local interaction energy and the repulsive energy maximum is the energy barrier $(\Delta E)$, which hinders deposited particles from leaving the primary minimum. Therefore, $\Delta E$ is regarded as an activation energy for particle release ${ }^{33}$. Once escaped from the primary minimum, depending on local thermal conditions, particles may escape from the secondary minimum, becoming free particles that transport along with the fluid stream.

To quantify the impact of the activation energy on $v_{\mathrm{pL}}$, we adopt the Arrhenius Equation ${ }^{23}$, i.e.,

$$
v_{\mathrm{pL}}=v_{0} \exp \left(-\frac{\Delta E}{k_{\mathrm{B}} T}\right),
$$

where $v_{0}$ is the escaping velocity when the energy barrier is absent, i.e., $\Delta E=0$.

In a realistic porous medium, where the ion distribution on the pore wall and the particles' instantaneous positions are inhomogeneous, the probability distribution of $\Delta E$ matters ${ }^{34,35}$. Considering a typical disordered medium obtained by randomly packed spheres, $\Delta E$ can be described by a Boltzmann distribution ${ }^{22}$, i.e., $f_{E}(\Delta E)=$ $\left(k_{\mathrm{B}} T_{0}\right)^{-1} \exp \left(-\Delta E / k_{\mathrm{B}} T_{0}\right)$, where $T_{0}$ is the temperature that represents the mean $\langle\Delta E\rangle=\int_{0}^{+\infty} \Delta E f_{E}(\Delta E) \mathrm{d} \Delta E=k_{\mathrm{B}} T_{0}$.
Based on $f_{V}\left(v_{\mathrm{pL}}\right)=f_{E}(\Delta E)\left|\mathrm{d} \Delta E / \mathrm{d} v_{\mathrm{pL}}\right|^{36}$, we derive the velocity PDF of particles near the pore wall as $f_{V}\left(v_{\mathrm{pL}}\right)=$ $\lambda v_{\mathrm{pL}}^{\lambda-1} / v_{0}^{\lambda}$, where $0 \leq v_{\mathrm{pL}} \leq v_{0}$ and $\lambda=T / T_{0}$.

Considering that particles, if not pre-existent in situ, must have transported to the near-wall region, the velocity probability of particles near the wall is a conditional probability given that event of particles reaching the near-wall region has occurred (whose probability is denoted by $\mathcal{P}$ ). Therefore, the conditional probability is $f_{V \mid \mathcal{P}}\left(v_{\mathrm{pL}}\right)=f_{V}\left(v_{\mathrm{pL}}\right) / \mathcal{P}$, where we model $\mathcal{P}$ by interception efficiency ${ }^{32,37}$ for non-pre-existent particles in Eq. (3) (also see SM, Sec. II), and $\mathcal{P}=1$ for pre-existent particles near the wall:

$\mathcal{P} \approx\left\{\begin{array}{lr}\left(\frac{d_{\mathrm{p}}}{l}\right)^{q}\left(\frac{\mathcal{D}_{\mathrm{w}} P e^{*}}{\kappa_{\mathrm{w}}}\right)^{2-q} & \text { (non-pre-existent particles) } \\ 1 & \text { (pre-existent particles) }\end{array}\right.$,

where $q=1.57$, and $\kappa_{\mathrm{w}}$ is kinematic viscosity of the ambient fluid.

By imposing $\int_{0}^{v_{0}} f_{V \mid \mathcal{P}}\left(v_{\mathrm{pL}}\right) \mathrm{d} v_{\mathrm{pL}}=1$, we obtain the conditional PDF of velocities near the pore wall:

$$
f_{V \mid \mathcal{P}}\left(v_{\mathrm{pL}}\right)=\frac{\alpha}{v_{0}^{\alpha}} v_{\mathrm{pL}}^{\alpha-1},
$$

where $\alpha=\lambda / \mathcal{P}=T / \mathcal{P} T_{0}$; the physical significance of $\alpha$ will be elaborated later.

Under the assumption that particles near the pore wall and near the pore center behave independently from each other at time $t \rightarrow+\infty$, we derive the joint PDF for the longitudinal velocity of the entire particle ensemble via Eqs. (1) and (4): $f_{V}\left(v_{\mathrm{pL}}\right)=\beta v_{\mathrm{pL}}^{\alpha-1} \exp \left(-\gamma v_{\mathrm{pL}}^{2}\right)$. Parameters $\beta$ and $\gamma$ are determined by imposing that $\int_{0}^{+\infty} f_{V}\left(v_{\mathrm{pL}}\right) \mathrm{d} v_{\mathrm{pL}}=1$ and $\int_{0}^{+\infty} f_{V}\left(v_{\mathrm{pL}}\right) v_{\mathrm{pL}}^{2} \mathrm{~d} v_{\mathrm{pL}}=\left\langle v_{\mathrm{pL}}^{2}\right\rangle$.

After defining a dimensionless velocity $v=v_{\mathrm{pL}} /\left\langle v_{\mathrm{w}}\right\rangle$, we derive (see SM, Sec. II) the velocity PDF $(\forall v \geq 0)$ for the entire particle ensemble:

$$
f_{V}(v)=\frac{2 m^{m}}{\Gamma(m) \Omega^{m}} v^{2 m-1} \exp \left(-\frac{m}{\Omega} v^{2}\right),
$$

where $m=\alpha / 2$ and $\Omega=\left\langle v^{2}\right\rangle$.

Equation (5) manifests as a Nakagami- $m$ distribution, in which the parameters $m$ and $\Omega$ are statistically referred to as shape and scale factors, respectively. The Nakagami- $m$ distribution was originally introduced to describe the fading signal intensity in wireless communications, which is characterized by $m \geq 0.5^{38}$. Because we show later that in particulate systems $m$ can be $<0.5$, we refer to Eq. (5) as a modified Nakagami-m distribution in the remainder of the Letter.

By letting $v \rightarrow+\infty$ and $v \rightarrow 0$, we find that the PDF in Eq. (5) for fast and slow particles is proportional to $\exp \left(-m v^{2} / \Omega\right)$ and $v^{2 m-1}$, respectively, which are proportional to Eqs. (1) and (4), respectively, indicating that the correspondent particles are those near the pore center and the pore wall, respectively. The rest of the particles, located between pore centers and walls, occupying a large portion of the entire ensemble, have intermediate velocities $(1<v<5$, see 

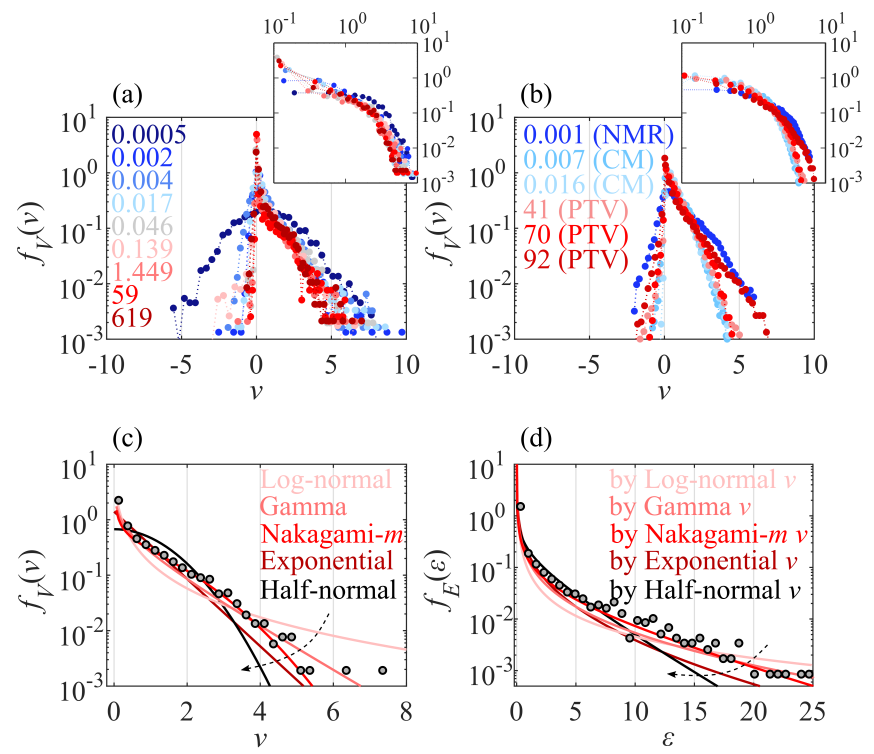

FIG. 1. (a) Probability distributions of $v$ as obtained by our Lagrangian simulations at $t=10 \tau$ under different $P e^{*}$ (see legend). The same distributions are presented in both semi-log and log-log plots (insets). (b) Experimental probability distributions of $v$ measured at different $\mathrm{Pe}^{*}$ via nuclear magnetic resonance $(\mathrm{NMR})^{20}$, confocal microscopy $(\mathrm{CM})^{10}$, and particle tracking velocimetry $(\mathrm{PTV})^{3}$ (see details about the experimental data in SM, Sec. XII). (c) ML estimations of the modified Nakagami- $m$ and other PDFs (see legend) based on the simulated $v$ data (symbols) at $P e^{*}=59$ (i.e., MP at $P e=1027$ ). (d) PDF predictions of $\varepsilon$ by the modified Nakagami- $m$ versus other $v$ PDFs (see legend) are compared to simulated $\varepsilon$ data (symbols) at $P e^{*}=59$ (i.e., $\mathrm{MP}$ at $P e=1027$ ).

SM, Sec. IX) that represent transition states from immobile (i.e., slow) to highly mobile (i.e., fast). Based on our analysis, their velocity distributions are not asymptotic to $v^{2 m-1}$ nor to $\exp \left(-m v^{2} / \Omega\right)$, but to the modified Nakagami- $m$ PDF (see SM, Sec. IX). Therefore, the modified Nakagami- $m$ distribution appears to be a unique, all-inclusive function for describing particle kinetics.

To assess both the uniqueness and the universality of the modified Nakagami- $m$ distribution (Eq. (5)), we perform numerical simulations of particles transport through a threedimensional randomly jammed packing of spheres (obstacles). The transporting particles considered include uranine (solute), nanoparticles (NPs), and microparticles (MPs). The relative importance of advection versus diffusion in establishing the particulate flow distribution and the fluid flow distribution are summarized by the particle Péclet number $P e^{*}=$ $\left\langle v_{\mathrm{w}}\right\rangle d_{\mathrm{p}} / \mathcal{D}_{\mathrm{w}}{ }^{26}$, and the pore Péclet number $P e=\left\langle v_{\mathrm{w}}\right\rangle l / \mathcal{D}_{\mathrm{w}}{ }^{39}$ (where $l$ is average pore size), respectively. In our numerical simulations, particles are subject to hydrodynamic drag, diffusive, and gravity (buoyancy) forces, and particle-wall interactions. In each simulation, 3000 particles of the same type are studied. Computational details, including the validation of the algorithm, are presented in SM, Secs. IV through $\mathrm{VII}^{40-48}$.

We obtain the probability data (PD) for simulated $v$ by sampling the entire ensemble of particles. To generate the PD for $t \rightarrow+\infty$ (the assumption for Eq. (1)), we analyze the simulated PD at $t=10 \tau$ for $5 \times 10^{-4} \leq P e^{*} \leq 619$, as shown in Figs. 1(a) and 2(a), where $\tau=l /\left\langle v_{\mathrm{w}}\right\rangle$ yields the characteristic advection time for fluid transport through an average pore size $l$. As $P e^{*}$ increases, the probability distributions transition from Gaussian to non-Gaussian, the latter of which features a sharp peak at near-zero velocities and heavy right tails. The numerical results agree with the experiments shown in Fig. 1(b). Based on the maximum-likelihood (ML) estimation, the tail over positive $v$ is fitted with the modified Nakagami- $m$ PDF and other widely used PDFs, e.g., exponential, half-normal, log-normal, and gamma distributions ${ }^{49}$. To fit the modified Nakagami- $m$ distribution, parameters $m$ and $\Omega$ are obtained by solving the derivatives of the logarithmic likelihood function ${ }^{50}$. For all cases studied when $P e^{*} \in\left[5 \times 10^{-4}, 619\right]$, out of all the functions considered, the modified Nakagami- $m$ distribution is the one most consistent with the entire range of velocity data, up to the noise floor. An example shown in Fig. 1(c) at $P e^{*}=59$ along with the cases at other $P e^{*}$ values (in $\mathrm{SM}, \mathrm{Sec}$. X) strongly supports the universality of Eq. (5).

Based on Eq. (5), we also derive the PDF of the dimensionless total energy $\varepsilon=\chi v^{2}$, where $\chi=\rho_{\mathrm{p}} / \rho_{\mathrm{w}}$ is the particle specific gravity (see definition of $\varepsilon$ and the derived $\varepsilon$ PDF in SM, Sec. III). We use the $m$ and $\Omega$ values, estimated from the ML Nakagami- $m$ fit of $v$ data, as inputs for the $\varepsilon$ PDF. The derived $\varepsilon$ PDF is then compared against its counterpart obtained computationally for all $P e^{*}$. A similar procedure is performed to derive other $\varepsilon$ PDFs by imposing different $v$ PDFs, and to compare their $\varepsilon$ predictions with the simulation data. Without tuning parameters, good agreement between the newly derived $\varepsilon$ PDF and the simulation data. This agreement demonstrates the predictive ability of Eq. (5) (more details in SM, Sec. XI).

Analysis of Eq. (5) shows that the scale parameter $\Omega=$ $\left\langle v^{2}\right\rangle=\left\langle v_{\mathrm{pL}}^{2}\right\rangle /\left\langle v_{\mathrm{w}}^{2}\right\rangle$ is related to the mean dimensionless total energy $\langle\varepsilon\rangle$, i.e., the mean total energy of particles compared to that of the ambient fluid, as $\Omega=\langle\varepsilon\rangle / \chi$. We, therefore, suggest that $\Omega$ is a transport parameter that reflects particles' transport strength. Our simulation results show that when the particles are initially introduced into the media, they acquire energy from the ambient fluid; consequently, $\Omega$ increases during the first characteristic advection time $(0<t \lesssim \tau)$. Thereafter, the driving force induced by the pressure gradient compensates the drag force to reach steady states. The particles at high $P e^{*}\left(\right.$ when $v_{\mathrm{pL}}=u_{\mathrm{pL}}$ and $w_{\mathrm{pL}}=0$ ) quickly reach these conditions, so that $v_{\mathrm{pL}} \rightarrow\left\langle v_{\mathrm{w}}\right\rangle$ and $\Omega \rightarrow 1$. Reaching such steady-state conditions can be delayed at lower $P e^{*}$, because diffusion dominates (when $v_{\mathrm{pL}}=w_{\mathrm{pL}}$ and $u_{\mathrm{pL}}=0$ ) and the correspondent disordered motion precludes $\Omega$ from reaching unity (see the simulated evolution of $\Omega$ with time in SM, Sec. VIII). Consistent with this analysis, both simulated and experimental data show that $\Omega$ at low $P e^{*}$ is higher than $\Omega$ at high $P e^{*}$ (see Fig. 2(b)).

In the original Nakagami- $m$ distribution, the shape parameter $m$ was statistically defined as $m=\left(\left\langle v^{2}\right\rangle / \sigma\left[v^{2}\right]\right)^{238}(\sigma[\cdot]$ is standard deviation), which makes itself relevant to the flatness of the PDF $\left(F=\left\langle v^{4}\right\rangle /\left\langle v^{2}\right\rangle^{251}\right)$ via $m=1 /(F-1)$. Be- 

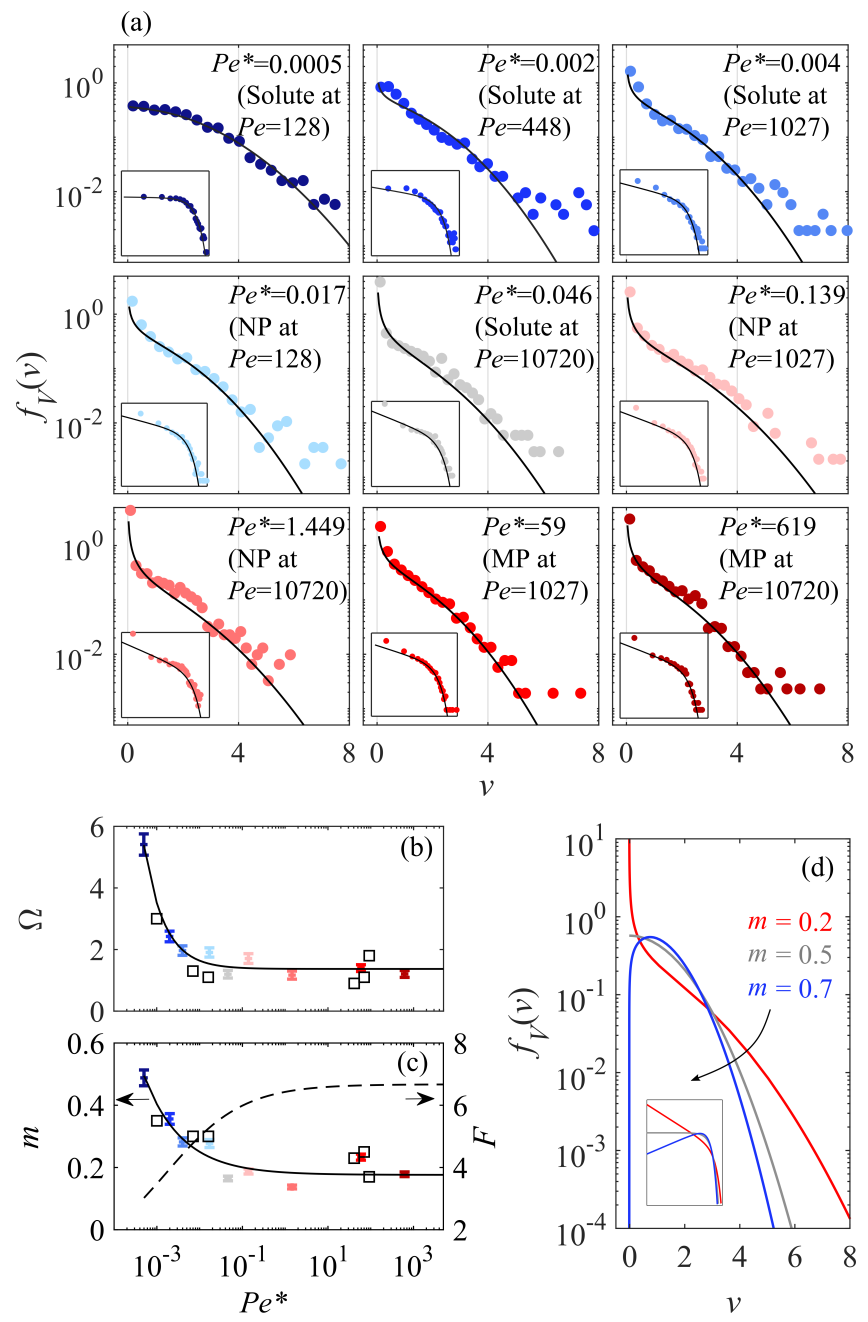

FIG. 2. (a) Modified Nakagami- $m$ fit (solid line) of $v$ data (symbols) from Lagrangian simulations at increasing $P e^{*} \in\left[5 \times 10^{-4}, 619\right]$ (The simulation data are the rescaled probabilities of positive $v$ in Fig. 1(a)). Estimates of (b) $\Omega$ and (c) $m$ from simulations (symbols with $95 \%$ confidence interval error bars) and experiments (squares) from Fig. 1(b). The power-law scaling curves (solid lines) are obtained by fitting simulation data as a function of $P e^{*}: \Omega=$ $0.004239 \times\left(P e^{*}\right)^{-0.9019}+1.37$ and $m=0.007877 \times\left(P e^{*}\right)^{-0.4867}+$ 0.1763 . The experimental data agree well with the anticipated powerlaw relations. $F$ is plotted as $F=1 / m+1$ (dashed line) where $m$ values are estimated from the power-law scaling of $m$ in (c). (d) The modified Nakagami- $m$ distribution reflects the net deposition which underscores the competition between the most probable diffusive energy and energy barrier for deposition. Insets in (a) and (d) are log$\log$ plots of the same respective distributions.

cause (1) $F$ can indicate the deviation from the Gaussian distribution $^{51,52}$, reflecting the degree of bend in a PDF curve at low velocities (e.g., $F=3$ and $m=0.5$ representing a Gaussian distribution), and (2) such velocities reflect the particles that may deposit near the wall, $F$ directly and $m$ inversely reflect the likelihood of particle deposition, i.e., $m$ is a deposition parameter.

Via the relation $m=\alpha / 2=T / 2 \mathcal{P} T_{0}$, derived in Eqs. (4) and (5), we find that the deposition strength $(\sim 1 / \mathrm{m})$ depends on $(1)$ the probability $(\mathcal{P})$ that particles will reach the nearwall region and (2) the temperature ratio $\left(T / T_{0}\right)$ near the pore wall. In other words:

(1) $m$ is a function of $\mathcal{P}$ in Eq. (3), i.e., pore structure (e.g., average pore size, $l$ ) and hydrodynamic conditions (e.g., $\left.P e^{*}\right)$. With the decrease of $l$, the pore space is more crowded, and thus more particles will reach the near-wall regions (i.e., $\mathcal{P}$ increases), promoting the particles' deposition (i.e., $m$ decreases) near the upstream spheres (see SM, Fig. S4(b)).

With the increase of $P e^{*}$, the likelihood of interception increases $^{32}$, i.e., the probability $\mathcal{P}$ increases, therefore deposition strength increases (see SM, Fig. S4(a)) and $m$ decreases. Via Eq. (3) and $m=T / 2 \mathcal{P} T_{0}$, we find that $m \propto\left(P e^{*}\right)^{-0.43}$. Such scaling coincides with the fitted function of the simulated $m$, as $m \propto\left(P e^{*}\right)^{-0.4867}$ in Fig. 2(c), which validates our theory for particles near the pore wall. Note that both theoretical and simulated $m$ values indicate that when $P e^{*} \geq 10, m$ reaches a plateau, indicating the deposition of the maximum strength has occurred once $P e^{*}$ reaches an intermediate value.

(2) $m$ also depends on $T / T_{0}$. The temperature $T$ is related to the kinetic energy of a particle with the most probable diffusive speed $\left(\hat{w}_{p}\right)$ in the MB distribution, i.e., the most probable diffusive energy: $\hat{K}_{\mathrm{D}}=m_{\mathrm{p}} \hat{w}_{\mathrm{p}}^{2} / 2=k_{\mathrm{B}} T$ (see SM, Sec. II). $T_{0}$ is related to the mean energy barrier, i.e., $\langle\Delta E\rangle=$ $k_{\mathrm{B}} T_{0}$ (see discussion related to $\Delta E$ distribution). Therefore, $m=\hat{K}_{\mathrm{D}} / 2 \mathcal{P}\langle\Delta E\rangle$, which indicates $m$ evaluates the competition between the most probable diffusive energy and the mean energy barrier due to the XDLVO interactions. Consider $\mathcal{P}=1$, i.e., under the circumstances that all particles were originally near the pore walls: As shown in Fig. 2(d), when $m<0.5, \hat{K}_{\mathrm{D}}<\langle\Delta E\rangle$, a net particle deposition occurs; when $m>0.5, \hat{K}_{\mathrm{D}}>\langle\Delta E\rangle$, the frequency of particles' release is greater than that of deposition, yielding zero net deposition; when $m=0.5, \hat{K}_{\mathrm{D}}=\langle\Delta E\rangle$, a kinetic equilibrium is achieved between the particles' deposition and release. In the last case, when $m=0.5$, the modified Nakagami- $m$ distribution reduces to the one-dimensional MB distribution $(\forall v \geq 0)$, we, therefore, suggest that the MB distribution holds not only for non-interacting particles, as traditionally believed, but also for charged particles when $\hat{K}_{\mathrm{D}}=\langle\Delta E\rangle$ and $\mathcal{P}=1$ both occur.

Non-Gaussian distributions $(m \neq 0.5)$ have been traditionally attributed to fluid stagnation and are known to be pronounced in heterogeneous media $11,14,39,53$. We find here that for transport with suspended particles, non-Gaussian distributions are indeed due to particle depositions in fluid stagnation regions; such deposition processes can be facilitated by factors other than pore structures, such as hydrodynamics (high $P e^{*}$ ) and particle-wall interactions (low $T / T_{0}$ ).

A putative universal probability distribution function is derived to describe for particles' kinetics in disordered porous media. The function reveals that non-Gaussian velocity distributions are a direct consequence of particles' transport and deposition, where their transport (reflected by $\Omega$ ) depends on $P e^{*}$ and their deposition (reflected by $m$ ) depends on $P e^{*}$, pore structure, and the energy imbalance near pore walls. The function's universality is predicated on the basis of the theoretical description of particle's hydrodynamic interception and trans- 
port, and electrokinetic release, and is further underpinned by Lagrangian particle-tracking simulations and experimental data. The uniqueness of the function consists in its predictive ability to describe the entire ensemble including immobile through highly mobile particles. Because our analysis of particle-wall interactions is applicable to particle-particle ones, the function is generalizable to other particulate systems where different interplays of particles and media arise, e.g., clustered granular gases that exhibit non-Gaussian kinetic distributions ${ }^{1,54-57}$.

Because the function proposed here accounts for phenomena such as particle size, flow rate, ion strength, it may help explain the synergy between interaction energy, hydrodynamics, and self-diffusion in controlling experimental particles deposition patterns. For example: (1) Hydrodynamics, represented by the particle Péclet number, has a positive impact on particles deposition, as discussed in Sec. V of the SM; this effect in our analysis is qualitatively consistent with direct experimental confocal microscopy observations ${ }^{58}$. (2) Because the proposed function considers particle-medium interactions via the XDLVO theory, it may help to interpret the progressive attachment of particles on medium surfaces when ionic conditions intensify ${ }^{59}$ or the enhanced deposition observed as a function of particles surface charge ${ }^{58}$. (3) Our simulation results also demonstrate a descending deposition pattern along with the porous medium, as discussed in Sec. V of SM, in qualitative agreement with confocal microscopy ${ }^{58}$ and coreflooding experiments on sand/bead packs ${ }^{60}$, indicating the applicability of our approach to designing experiments of particulate flows.

\section{SUPPLEMENTARY MATERIAL}

In Supplementary Material, we provide nomenclature (Sec. I), a detailed derivation of the universal function, Eq. (5) (Sec. II), a summary of the derived energy probability distribution functions (Sec. III), details of numerical simulations (Sec. IV), numerical analysis of deposited particles (Sec. V), statistical check of particle numbers (Sec. VI), validation of the numerical simulations (Sec. VII), the evolution of $\Omega$ with time (Sec. VIII), analysis of slow, intermediate, and fast particles (Sec. IX), maximum-likelihood estimation of velocity probability distributions (Sec. X), predictions of energy distributions for different velocity probability distributions (Sec. XI), and discussions about the experimental data used in this work (Sec. XII).

\section{ACKNOWLEDGMENTS}

This work is supported by the Science4CleanEnergy European research consortium funded by European Union's Horizon 2020 research and innovation programme, under Grant Agreement No. 764810 (S4CE). Generous allocations of computing time were provided by the University College London Research Computing Platforms Support (Kathleen). We are indebted to M. Holzner and M. Carrel for offering three- dimensional particle tracking velocimetry experimental data as well as V.L. Morales for helpful correspondence.

\section{DATA AVAILABILITY}

The data that support the findings of this study are available from the corresponding author upon reasonable request.

\section{REFERENCES}

${ }^{1}$ J. S. van Zon and F. C. MacKintosh, Physical Review Letters 93, 038001 (2004).

${ }^{2}$ F. Höfling, T. Franosch, and E. Frey, Physical Review Letters 96, 165901 (2006).

${ }^{3}$ M. Carrel, V. L. Morales, M. Dentz, N. Derlon, E. Morgenroth, and M. Holzner, Water Resources Research 54, 2183 (2018).

${ }^{4}$ P. K. Kang, P. de Anna, J. P. Nunes, B. Bijeljic, M. J. Blunt, and R. Juanes, Geophysical Research Letters 41, 6184 (2014).

${ }^{5} \mathrm{~N}$. Tufenkji and M. Elimelech, Environmental Science \& Technology 38, 529 (2004).

${ }^{6}$ S. A. Bradford, J. Simunek, M. Bettahar, M. T. van Genuchten, and S. R. Yates, Environmental Science \& Technology 37, 2242 (2003).

${ }^{7}$ S.-J. Yook and K.-H. Ahn, Applied Physics Letters 94, 191909 (2009).

${ }^{8}$ I. Tuval, I. Mezić, F. Bottausci, Y. T. Zhang, N. C. MacDonald, and O. Piro, Physical Review Letters 95, 236002 (2005).

${ }^{9}$ M. Eglin, M. A. Eriksson, and R. W. Carpick, Applied Physics Letters 88, 091913 (2006).

${ }^{10}$ S. S. Datta, H. Chiang, T. S. Ramakrishnan, and D. A. Weitz, Physical Review Letters 111, 064501 (2013).

${ }^{11}$ M. Siena, M. Riva, J. D. Hyman, C. L. Winter, and A. Guadagnini, Physical Review E 89, 013018 (2014).

${ }^{12}$ M. Matyka, J. Gołembiewski, and Z. Koza, Physical Review E 93, 013110 (2016).

${ }^{13}$ S. Aramideh, P. P. Vlachos, and A. M. Ardekani, Physical Review E 98, 013104 (2018).

${ }^{14}$ P. de Anna, B. Quaife, G. Biros, and R. Juanes, Physical Review Fluids 2, 124103 (2017).

${ }^{15}$ P. de Anna, T. Le Borgne, M. Dentz, A. M. Tartakovsky, D. Bolster, and P. Davy, Physical Review Letters 110, 184502 (2013).

${ }^{16}$ K. Alim, S. Parsa, D. A. Weitz, and M. P. Brenner, Physical Review Letters 119, 144501 (2017).

${ }^{17}$ F. Rouyer, J. Martin, and D. Salin, Physical Review Letters 83, 1058 (1999).

${ }^{18}$ G. R. Guédon, F. Inzoli, M. Riva, and A. Guadagnini, Physical Review E 100, 043101 (2019).

${ }^{19}$ K.-S. Im, K. Fezzaa, Y. Wang, X. Liu, J. Wang, and M.-C. Lai, Applied Physics Letters 90, 091919 (2007).

${ }^{20}$ Y. Kutsovsky, L. Scriven, H. Davis, and B. E. Hammer, Physics of Fluids 8, 863 (1996).

${ }^{21}$ B. Berkowitz, A. Cortis, M. Dentz, and H. Scher, Reviews of Geophysics 44 (2006).

${ }^{22}$ D. A. McQuarrie and J. D. Simon, Physical chemistry: A molecular approach (Sterling Publishing Company, 1997).

${ }^{23}$ R. Kretzschmar, M. Borkovec, D. Grolimund, and M. Elimelech, Mobile subsurface colloids and their role in contaminant transport, in Advances in agronomy, Vol. 66 (Elsevier, 1999) pp. 121-193.

${ }^{24} \mathrm{H}$. Brenner, Philosophical Transactions of the Royal Society of London. Series A, Mathematical and Physical Sciences 297, 81 (1980).

${ }^{25}$ Y. Klimontovich, Statistical Theory of Open Systems: Volume 1: A Unified Approach to Kinetic Description of Processes in Active Systems, Fundamental Theories of Physics (Springer Netherlands, 1995).

${ }^{26}$ D. Kandhai, D. Hlushkou, A. G. Hoekstra, P. M. A. Sloot, H. Van As, and U. Tallarek, Physical Review Letters 88, 234501 (2002).

${ }^{27} \mathrm{~W}$. Pauli and C. P. Enz, Thermodynamics and the kinetic theory of gases, Vol. 3 (Courier Corporation, 2000). 
${ }^{28}$ A. A. Schekochihin, Lectures on Kinetic Theory of Gases and Statistical Physics (University of Oxford, 2018).

${ }^{29}$ F. Civan, Porous Media Transport Phenomena (John Wiley \& Sons, 2011).

${ }^{30}$ P. L. Bowers, Lectures on Quantum Mechanics: A Primer for Mathematicians (Cambridge University Press, 2020).

${ }^{31}$ F. Schwabl and W. Brewer, Statistical Mechanics, Advanced Texts in Physics (Springer Berlin Heidelberg, 2006).

${ }^{32}$ J. H. Masliyah and S. Bhattacharjee, Electrokinetic and colloid transport phenomena (John Wiley \& Sons, 2006).

${ }^{33}$ T. Bhattacharjee and S. S. Datta, Nature Communications 10, 1 (2019).

${ }^{34}$ C. Shen, Y. Jin, J. Zhuang, T. Li, and B. Xing, Critical Reviews in Environmental Science and Technology 50, 244 (2020).

${ }^{35}$ D. Grolimund and M. Borkovec, Environmental Science \& Technology 33, 4054 (1999).

${ }^{36}$ D. C. Montgomery and G. C. Runger, Applied Statistics and Probability for Engineers (Wiley, 2003).

${ }^{37}$ M. Weber and D. Paddock, Journal of Colloid and Interface Science 94, 328 (1983).

${ }^{38} \mathrm{M}$. Nakagami, The $m$-distribution-a general formula of intensity distribution of rapid fading, in Statistical methods in radio wave propagation (Elsevier, 1960) pp. 3-36.

${ }^{39}$ B. Bijeljic, P. Mostaghimi, and M. J. Blunt, Physical Review Letters 107, 204502 (2011).

${ }^{40}$ D. Seguin, A. Montillet, and J. Comiti, Chemical Engineering Science 53, 3751 (1998).

${ }^{41}$ M. Skoge, A. Donev, F. H. Stillinger, and S. Torquato, Physical Review E 74, 041127 (2006).

${ }^{42}$ H. Zhang, J. Pan, X. He, and M. Pan, Journal of Applied Polymer Science 107, 3306 (2008).

${ }^{43}$ V. L. Morales, M. Dentz, M. Willmann, and M. Holzner, Geophysical Research Letters 44, 9361 (2017).
${ }^{44}$ H. Ma, J. Pedel, P. Fife, and W. P. Johnson, Environmental Science \& Technology 43, 8573 (2009).

${ }^{45}$ G. Mikutis, C. A. Deuber, L. Schmid, A. Kittilä, N. Lobsiger, M. Puddu, D. O. Asgeirsson, R. N. Grass, M. O. Saar, and W. J. Stark, Environmental science \& technology 52, 12142 (2018).

${ }^{46}$ J. Happel and H. Brenner, Low Reynolds number hydrodynamics: with special applications to particulate media, Vol. 1 (Springer Science \& Business Media, 2012).

${ }^{47}$ M. Holzner, V. L. Morales, M. Willmann, and M. Dentz, Physical Review E 92, 013015 (2015).

${ }^{48}$ C. Asbach, H. Fissan, T. A. Kuhlbusch, J. Wang, and D. Y. Pui, Applied Physics Letters 93, 054104 (2008).

${ }^{49} \mathrm{~F}$. James, Statistical methods in experimental physics (World Scientific Publishing Company, 2006).

${ }^{50}$ J. Cheng and N. C. Beaulieu, IEEE Communications Letters 5, 101 (2001).

${ }^{51}$ J. S. Olafsen and J. S. Urbach, Physical Review E 60, R2468 (1999).

${ }^{52}$ G. Baxter and J. S. Olafsen, Nature 425, 680 (2003).

${ }^{53}$ M. Dentz, M. Icardi, and J. J. Hidalgo, Journal of Fluid Mechanics 841, 851 (2018).

${ }^{54}$ K. Harth, T. Trittel, S. Wegner, and R. Stannarius, Physical Review Letters 120, 214301 (2018).

${ }^{55}$ A. Prevost, D. A. Egolf, and J. S. Urbach, Physical Review Letters 89, 084301 (2002).

${ }^{56}$ J. S. Olafsen and J. S. Urbach, Physical Review Letters 81, 4369 (1998).

${ }^{57}$ P. Yu, M. Schröter, and M. Sperl, Physical Review Letters 124, 208007 (2020).

${ }^{58}$ N. Bizmark, J. Schneider, R. D. Priestley, and S. S. Datta, Science Advances 6, eabc2530 (2020).

${ }^{59}$ G. Gerber, M. Bensouda, D. A. Weitz, and P. Coussot, Physical Review Letters 123, 158005 (2019).

${ }^{60}$ W. P. Johnson, Environmental Science \& Technology 54, 8032 (2020). 